\title{
Level of physical exercises' mastering in structure of 11-13 yrs age boys' motor fitness
}

\author{
Ivashchenko O.V. ${ }^{1}$, Iermakov S.S. ${ }^{2}$, Khudolii O.M. ${ }^{1}$, Cretu M. ${ }^{3}$, Potop V. \\ ${ }^{1}$ H.S. Skovoroda Kharkiv National Pedagogical University, Ukraine \\ ${ }^{2}$ Kazimierz Wielki University, Bydgoszcz, Poland \\ ${ }^{3}$ University of Pitesti, Romania \\ ${ }^{4}$ Ecological University of Bucharest, Romania
}

\begin{abstract}
Purpose:

Material:

to find the place of physical exercises' mastering level in structure of 11-13 yrs age boys' motor fitness.

in the research 11 yrs age boys $(n=58), 12(n=76)$ and 13 years age $(n=93)$ participated. Testing program included well-known tests: "Forward roll", "Backward roll", "Vault", "Climbing rope (three attempts)", "Bridge”, "Stance on shoulder blades".

Results: $\quad$ By indicators of physical condition 11-13 yrs age boys statistically confidently differ one from another $(p<0,001)$. Level of mastering of gymnastic exercises "Backward roll,, "Vault", "Climbing rope (three attempts)", "Bridge", "Stance on shoulder blades" with age statistically confidently increases $(p<0,001)$.

Conclusions: In factorial structure of motor fitness, level of physical exercises' mastering has weight 17,5\% (11 yrs age), $36,6 \%$ (12 years), $28,5 \%$ (13 years). Analysis of communities showed that in 11-13 yrs age boys training of motor abilities is effective (if they become a component of mastered motor skills).

Keywords: motor abilities, level of mastering, factorial analysis, 11-13 yrs age boys.
\end{abstract}

\section{Introduction}

The problem of optimization of schoolchildren's physical education was regarded in works of Bodnar I. [1], Vas'kov Iu.V. [2], Krucevich T. et al. [9], Ivashchenko O.V. [8]. Bodnar I. stresses on need in searching new approaches to integrated physical education of different health groups' schoolchildren [1]. The authors defined urgent problems of educational process's perfection at physical culture lessons. Theoretical aspects and conditions of implementation in educational process of such innovative approaches as culturologic, competence, synergetic, axiologic, achmeologic and other are regarded. The ways of the mentioned approaches' implementation in real educational process are open. The results of innovative approaches implementation in personality oriented educational process are analyzed. It was proved that implementation of innovative approaches facilitates rising of educational process's organization. Its basis is personality oriented approach to pupils. Besides, certain difficulties in educational process's organization in comprehensive schools were found [2]. Methodic materials for planning of physical education process in comprehensive schools have been worked out [9]. Conception of physical education, which was built on the base of physical education's simulation, motor abilities' training and pedagogic control has been created. This conception includes: application of factorial and discriminant models of functional state age changes and motor fitness. The purpose of this conception is: planning of educational material, current, finalizing and stage-bystage control of children's fitness; modes of loading in a lesson and in series of lessons; regimes of alternation of relaxation and exercises [8].

In schoolchildren's physical education there marked

(c) Ivashchenko O.V., lermakov S.S., Khudolii O.M.,

Cretu M., Potop V., 2017

doi:10.15561/18189172.2017.0506 out directions of researches, which are connected with studying of motor abilities $[5,6,7]$ and process of motor actions' training $[17,20,28]$. Peculiarities of functional, coordination and power fitness of children and adolescents have been found $[4,11]$. Dependence of training of power loads' effects on regime of exercises' fulfillment and relaxation has been determined [18, 23]. The process of motor actions' training was studied from position of interdisciplinary connections $[12,13]$; formation of motor competence $[14,15]$; formation of meta-cognitive behavior [16]; verbal perception in mastering of sport movements [19]; optimization of regimes of exercises' repetitions and rest intervals [20, 26, 28].

However, in available scientific literature there is a little data about wholeness of motor abilities' development and children's and adolescents' training [27, 29]. Thus, study of motor fitness influence on level of physical exercises' mastering is rather relevant. In other works training of motor fitness and dynamic of physical exercises' mastering in 11-13 yrs age girls were regarded $[31,32]$. In our work we have studied motor fitness and dynamic of physical exercises' mastering in 11-13 yrs age boys.

The purpose of the research is to find the place of physical exercises' mastering level in structure of 11-13 yrs age boys' motor fitness.

\section{Material and methods}

Participants: in the research 11 yrs age boys $(\mathrm{n}=58)$, $12(n=76)$ and 13 years age $(n=93)$ participated.

Organization of the research: we used the following methods of research: analysis of scientific-methodic literature, pedagogic testing, methods of mathematical statistic. Testing program included well known tests. We registered: body length and mass, vital capacity of lungs (VCL), right and left hand dynamometry. We registered 
results in the following tests: "Pressing ups", times", "legs' rising in hanging on Sweden wall position, times", "Angle on parallel bars, sec.", "Torso rising in sitting position from lying on back position during 1 min.", "Forward torso rising from sitting position (legs apart), cm", "Hanging on bent arms, sec.", "'Torso rising from lying on abdomen position during $30 \mathrm{sec}$., times", "Long jump from the sport, cm,", "Throw of filled ball $(1 \mathrm{~kg})$ in sitting position", "Shuttle run $4 \times 9 \mathrm{~m}$, sec." [25].

We studied mastering level of exercises: "Forward roll", "Backward roll", "Vault", "Climbing rope (three attempts)", "Bridge", "Stance on shoulder blades". [27].

Statistical analysis: the data were processed with the help of statistical analysis program IBM SPSS 20 . The used factorial analysis included implied method of principle components. Method of rotation implied Varimax with normalization of Keiser. For every variable we calculated the following statistics: mean values,standard deviations, Student's t-test for independent samples. The level of mastering was determined as correlation of successful attempts $(\mathrm{m})$ and their general quantity $(5): \mathrm{p}=(\mathrm{m} / 5)$ $\mathrm{x} 100$.

\section{Results}

Results of the researches are presented in tables 1-7. By indicators of physical condition 11-13 yrs age boys confidently differ one from another $(\mathrm{p}<0,001)$. Body length increased by $8,6 \%$, by $30,9 \%$ — body mass, by $32,33 \%$ - vital capacity of lungs, by $39,7 \%$ — strength of right hand and by $38,3,8 \%$ - strength of left hand.

12 yrs boys show confidently better results than 11 yrs boys in tests: "Chin ups in lying position, times", "Legs' rising, hanging on Sweden wall, times", "Angle on parallel bars, sec., times", "Forward torso bending in sitting position (legs apart), cm", "Hanging on bent arms, sec.", "Torso rising from lying on abdomen position during $30 \mathrm{sec}$., times", "Long jump from the spot, cm", "Throw of filled ball $(1 \mathrm{~kg})$ from sitting position, $\mathrm{cm}$ ".

13 yrs boys show confidently better results in the following tests: "Pressing ups, times", "Chin ups, times", "Legs' rising, hanging on Sweden wall, times", "Angle on parallel bars, sec., times", "Torso rising from position lying on back during 1 minute, times", "Torso rising from lying on abdomen position during $30 \mathrm{sec}$., times", "Long jump from the spot, cm", "Throw of filled ball (1 kg) from

Table 1. Testing results of $11-13$ yrs boys

\begin{tabular}{|c|c|c|c|c|c|c|c|c|}
\hline $\begin{array}{l}\text { № } \\
\text { № }\end{array}$ & Description of parameters & Age & $\mathbf{N}$ & $x$ & m & $\begin{array}{l}\text { Difference } \\
\text { of mean } \\
\text { values }\end{array}$ & $\mathbf{t}$ & $\mathbf{P}$ \\
\hline \multirow{4}{*}{1} & \multirow{3}{*}{ Height, cm } & 11 & 58 & 143,172 & 857 & $-7,182^{*}$ & $-5,654^{*}$ & $<0,001$ \\
\hline & & 12 & 76 & 150,355 & 896 & $-5,225^{* *}$ & $-4,581^{* *}$ & $<0,001$ \\
\hline & & 13 & 93 & 155,580 & ,726 & $-12,408^{* * *}$ & $-10,866^{* * *}$ & $<0,001$ \\
\hline & & 11 & 58 & 35,586 & ,955 & $-6,716$ & $-4,616$ & $<0,01$ \\
\hline \multirow[t]{3}{*}{2} & \multirow[t]{3}{*}{ Body mass, kg } & 12 & 76 & 42,302 & 1,040 & $-4,288$ & $-2,889$ & $<0,001$ \\
\hline & & 13 & 93 & 46,591 & 1,038 & $-11,005$ & $-7,257$ & $<0,001$ \\
\hline & & 11 & 58 & 1722,413 & 45,951 & $-285,480$ & $-4,904$ & $<0,01$ \\
\hline \multirow[t]{3}{*}{3} & \multirow[t]{3}{*}{$\mathrm{VCL}, \mathrm{cm} 3$} & 12 & 76 & 2007,894 & 36,838 & $-270,599$ & $-5,055$ & $<0,001$ \\
\hline & & 13 & 93 & 2278,494 & 37,880 & $-556,080$ & $-9,245$ & $<0,001$ \\
\hline & & 11 & 58 & 18,448 &, 360 & $-3,485$ & $-4,220$ & $<0,01$ \\
\hline \multirow[t]{3}{*}{4} & \multirow{3}{*}{ Right hand dynamometry, kg } & 12 & 76 & 21,934 & ,667 & $-3,850$ & $-4,031$ & $<0,001$ \\
\hline & & 13 & 93 & 25,784 & 670 & $-7,336$ & $-8,189$ & $<0,001$ \\
\hline & & 11 & 58 & 17,586 & ,646 & $-2,295$ & $-2,319$ & $<0,05$ \\
\hline \multirow[t]{3}{*}{5} & \multirow[t]{3}{*}{ Left hand dynamometry, kg } & 12 & 76 & 19,881 & ,710 & $-4,441$ & $-4,536$ & $<0,001$ \\
\hline & & 13 & 93 & 24,322 & ,668 & $-6,736$ & $-6,814$ & $<0,001$ \\
\hline & & 11 & 58 & 20,224 & 679 & $-1,657$ & $-1,416$ & $>0,05$ \\
\hline \multirow[t]{3}{*}{6} & \multirow[t]{3}{*}{ Pressing ups, times } & 12 & 76 & 21,881 & 881 & $-2,828$ & $-2,224$ & $<0,05$ \\
\hline & & 13 & 93 & 24,709 & 895 & $-4,485$ & $-3,572$ & $<0,001$ \\
\hline & & 11 & 58 & 1,758 & 184 &,- 438 & $-1,455$ & $>0,05$ \\
\hline \multirow[t]{3}{*}{7} & \multirow[t]{2}{*}{ Chin ups, times } & 12 & 76 & 2,197 & 222 & $-1,361$ & $-3,572$ & $<0,001$ \\
\hline & & 13 & 93 & 3,559 & ,292 & $-1,800$ & $-4,517$ & $<0,001$ \\
\hline & \multirow{3}{*}{$\begin{array}{l}\text { Legs' rising, hanging on } \\
\text { Sweden wall, times }\end{array}$} & 11 & 58 & 4,758 & ,394 &,- 0440 &,- 073 & $>0,05$ \\
\hline \multirow[t]{2}{*}{8} & & 12 & 76 & 4,802 & 431 & $-2,810$ & $-4,647$ & $<0,001$ \\
\hline & & 13 & 93 & 7,612 & , 417 & $-2,854$ & $-4,649$ & $<0,001$ \\
\hline \multirow{3}{*}{9} & \multirow{3}{*}{$\begin{array}{l}\text { Angle on parallel bars, sec., } \\
\text { times }\end{array}$} & 11 & 58 & 1,206 & 211 & 009 & 035 & $>0,05$ \\
\hline & & $\begin{array}{l}1 \\
12\end{array}$ & 76 & 1,197 & 177 &,- 609 & $-2,294$ & $<0,05$ \\
\hline & & 13 & 93 & 1,806 & - 191 & 599 & 2,039 & $<0,05$ \\
\hline \multirow{3}{*}{10} & Torso rising from position & 11 & 58 & 39,000 & ,736 & ,592 & 617 & $>0,05$ \\
\hline & \multirow{2}{*}{$\begin{array}{l}\text { lying on back during } 1 \text { minute, } \\
\text { times }\end{array}$} & 12 & 76 & 38,407 & 621 & $-5,151$ & $-5,615$ & $<0,001$ \\
\hline & & 13 & 93 & 43,559 & 655 & $-4,559$ & $-4,497$ & $<0,001$ \\
\hline \multirow{3}{*}{11} & \multirow{3}{*}{$\begin{array}{l}\text { Forward torso bending in } \\
\text { sitting position (legs apart), } \\
\mathrm{cm}\end{array}$} & 11 & 58 & 4,051 & ,380 & $-1,448$ & $-2,728$ & $<0,01$ \\
\hline & & 12 & 76 & 5,500 & ,361 & 2,231 & 5,305 & $<0,001$ \\
\hline & & 13 & 93 & 3,268 & ,239 & ,782 & 1,835 & $>0,05$ \\
\hline \multirow{3}{*}{12} & \multirow{3}{*}{ Hanging on bent arms, sec } & 11 & 58 & 5,327 & 692 & $-1,646$ & $-1,368$ & $>0,05$ \\
\hline & & 12 & 76 & 6,973 & ,907 & $-2,563$ & $-1,996$ & $<0,05$ \\
\hline & & 13 & 93 & 9,537 & 893 & $-4,210$ & $-3,348$ & $<0,001$ \\
\hline
\end{tabular}


Table 1 (Continued)

\begin{tabular}{|c|c|c|c|c|c|c|c|c|}
\hline $\begin{array}{l}\text { № } \\
\text { № }\end{array}$ & Description of parameters & Age & $\mathbf{N}$ & $\mathbf{X}$ & $\mathrm{m}$ & $\begin{array}{l}\text { Difference } \\
\text { of mean } \\
\text { values }\end{array}$ & $\mathbf{t}$ & $\mathbf{P}$ \\
\hline \multirow[t]{2}{*}{13} & $\begin{array}{l}\text { Torso rising from lying on } \\
\text { abdomen position during } 30\end{array}$ & $\begin{array}{l}11 \\
12\end{array}$ & $\begin{array}{l}58 \\
76\end{array}$ & $\begin{array}{l}19,137 \\
20,947\end{array}$ & $\begin{array}{l}399 \\
\text {,515 }\end{array}$ & $\begin{array}{l}-1,809 \\
-4,783\end{array}$ & $\begin{array}{l}-2,638 \\
-6,704\end{array}$ & $\begin{array}{l}<0,005 \\
<0,001\end{array}$ \\
\hline & sec., times & 13 & 93 & 25,731 & ,488 & $-6,593$ & $-9,494$ & $<0,001$ \\
\hline \multirow{3}{*}{14} & & 11 & 58 & 144,913 & 2,282 & $-5,217$ & $-1,831$ & $>0,05$ \\
\hline & Long jump from the spot, $\mathrm{cm}$ & 12 & 76 & 150,131 & 1,779 & $-16,513$ & $-7,678$ & $<0,001$ \\
\hline & & 13 & 93 & 166,645 & 1,290 & $-21,731$ & $-8,935$ & $<0,001$ \\
\hline \multirow{4}{*}{15} & Throw of filled ball $(1 \mathrm{~kg})$ from & 11 & 58 & 209,396 & 5,283 & $-57,313$ & $-6,838$ & $<0,001$ \\
\hline & Inrow of Tlllea dall (1 kg) trom & 12 & 76 & 266,710 & 6,109 & $-69,633$ & $-9,435$ & $<0,001$ \\
\hline & & 13 & 93 & 336,344 & 4,427 & $-126,947$ & $-18,163$ & $<0,001$ \\
\hline & & 11 & 58 & 11,667 & 101 & 140 & 1,016 & $>0,05$ \\
\hline \multirow[t]{2}{*}{16} & Shuttle run, $4 \times 9 \mathrm{~m}$, sec. & 12 & 76 & 11,526 & ,093 & 119 & 107 & $>0,05$ \\
\hline & & 13 & 93 & 11,406 & 1,010 & ,260 & ,203 & $>0,05$ \\
\hline \multirow{3}{*}{17} & Forward roll level of & 11 & 58 & 80,689 & 2,252 & $-9,047$ & $-3,126$ & $<0,002$ \\
\hline & Forward roll, level of & 12 & 76 & 89,736 & 1,854 & $-3,596$ & $-1,537$ & $>0,05$ \\
\hline & mastering & 13 & 93 & 93,333 & 1,477 & $-12,643$ & $-4,900$ & $<0,001$ \\
\hline \multirow{3}{*}{18} & Backward roll level of & 11 & 58 & 64,931 & 2,926 & $-14,016$ & $-3,557$ & $<0,001$ \\
\hline & Backwara roll, level ot & 12 & 76 & 78,947 & 2,619 & $-9,654$ & $-2,877$ & $<0,005$ \\
\hline & & 13 & 93 & 88,602 & 2,149 & $-23,671$ & $-6,631$ & $<0,001$ \\
\hline \multirow{3}{*}{19} & & 11 & 58 & 72,069 & 2,553 & $-18,983$ & $-6,362$ & $<0,001$ \\
\hline & Vault, level of mastering & 12 & 76 & 91,052 & 1,732 &,- 775 &,- 299 & $>0,05$ \\
\hline & & 13 & 93 & 91,828 & 1,865 & $-19,758$ & $-6,362$ & $<0,001$ \\
\hline \multirow{4}{*}{20} & & 11 & 58 & 68,620 & 3,482 & $-16,379$ & $-3,995$ & $<0,05$ \\
\hline & Cimoing rope (tnree & 12 & 76 & 85,000 & 2,402 & $-1,236$ &,- 335 & $>0,05$ \\
\hline & & 13 & 93 & 86,236 & 2,700 & $-17,615$ & $-4,015$ & $<0,001$ \\
\hline & & 11 & 58 & 82,069 & 3,432 & $-11,878$ & $-3,388$ & $<0,002$ \\
\hline \multirow[t]{2}{*}{21} & Bridge, level of mastering & 12 & 76 & 93,947 & 1,590 & 3,839 & 1,445 & $>0,05$ \\
\hline & & 13 & 93 & 90,107 & 2,019 & $-8,038$ & $-2,158$ & $<0,05$ \\
\hline \multirow{3}{*}{22} & & 11 & 58 & 85,517 & 2,243 & $-2,903$ &,- 986 & $>0,05$ \\
\hline & Stance on snoulder blades, & 12 & 76 & 88,421 & 1,919 & $-4,267$ & $-1,614$ & $>0,05$ \\
\hline & level of mastering & 13 & 93 & 92,688 & 1,803 & $-7,170$ & $-2,481$ & $<0,05$ \\
\hline
\end{tabular}

* comparison of 11-12 years; ** comparison of $12-13$ years; *** comparison of $11-13$ years

Table 2. Matrix of factorial analysis of 11 yrs boys' testing. Rotation method: Varimax with Keiser's normalization

\begin{tabular}{|c|c|c|c|c|c|c|c|c|}
\hline \multirow{2}{*}{$\begin{array}{l}\text { № } \\
\text { № }\end{array}$} & \multirow{2}{*}{ Description of parameters } & \multicolumn{6}{|c|}{ Component } & \multirow{2}{*}{ h2 } \\
\hline & & 1 & 2 & 3 & 4 & 5 & 6 & \\
\hline 1 & $\mathrm{VCL}, \mathrm{cm} 3$ & & ,778 & & ,379 & & & ,803 \\
\hline 2 & Right hand dynamometry, kg & & ,702 & ,343 & ,318 & & & ,827 \\
\hline 3 & Left hand dynamometry, kg & & & & 741 & & & 633 \\
\hline 4 & Pressing ups, times & &, 530 &,- 606 & & & & 784 \\
\hline 5 & Chin ups, times & & ,373 &,- 693 & & & & ,788 \\
\hline 6 & Legs' rising, hanging on Sweden wall, times & 706 & & & & & & ,577 \\
\hline 7 & Angle on parallel bars, sec., times & ,803 & & & & &,- 346 & ,897 \\
\hline 8 & $\begin{array}{l}\text { Torso rising from position lying on back during } 1 \\
\text { minute, times }\end{array}$ &, 534 & -472 & & ,326 & & & ,719 \\
\hline 9 & $\begin{array}{l}\text { Forward torso bending in sitting position (legs } \\
\text { apart), } \mathrm{cm}\end{array}$ & 777, & & & & & & ,706 \\
\hline 10 & Hanging on bent arms, sec & 664 &,- 329 & & & & 310 & ,747 \\
\hline 11 & $\begin{array}{l}\text { Torso rising from lying on abdomen position } \\
\text { during } 30 \text { sec., times }\end{array}$ & &,- 368 & & & 635, & & 639, \\
\hline 12 & Long jump from the spot, $\mathrm{cm}$ &, 569 &,- 382 & & & ,305 &,- 449 & ,854 \\
\hline 13 & $\begin{array}{l}\text { Throw of filled ball }(1 \mathrm{~kg}) \text { from sitting position, } \\
\mathrm{cm}\end{array}$ & ,725 & & & & & & ,602 \\
\hline 14 & Shuttle run, $4 \times 9 \mathrm{~m}$, sec. & 627 & & & & & &, 510 \\
\hline 15 & Forward roll, level of mastering & ,657 & & & & &,- 378 & 601 \\
\hline
\end{tabular}


Table 2 (Continued)

\begin{tabular}{|c|c|c|c|c|c|c|c|c|}
\hline \multirow{2}{*}{$\begin{array}{l}\text { № } \\
\text { № }\end{array}$} & \multirow{2}{*}{ Description of parameters } & \multicolumn{6}{|c|}{ Component } & \multirow{2}{*}{ h2 } \\
\hline & & 1 & 2 & 3 & 4 & 5 & 6 & \\
\hline 16 & Backward roll, level of mastering & & & ,446 & & ,615 & & 750 \\
\hline 17 & Vault, level of mastering & ,759 & & & & & & 685 \\
\hline 18 & $\begin{array}{l}\text { Climbing rope (three attempts), level of } \\
\text { mastering }\end{array}$ & ,778 & & & & & & ,750 \\
\hline 19 & Bridge, level of mastering & ,834 & & & & & & ,815 \\
\hline 20 & Stance on shoulder blades, level of mastering & 850 & & & & & & ,860 \\
\hline 21 & Bridge, level of mastering & ,532 & ,412 & 366 &,- 390 & & & ,759 \\
\hline 22 & Stance on shoulder blades, level of mastering & ,736 & & & & & & ,706 \\
\hline
\end{tabular}

Table 3. Full explained dispersion

\begin{tabular}{llll}
\hline \multirow{2}{*}{ Component } & Interpretation & \multicolumn{2}{c}{ Sum of squares of rotation loads } \\
& & Cof dispersion & Cumulative \% \\
\hline 1 & Level of mastering and motor fitness & 17,505 & 17,505 \\
2 & Physical condition & 17,108 & 34,613 \\
3 & Strength of hand & 13,390 & 48,003 \\
4 & Functional state of respiratory system & 8,954 & 56,957 \\
5 & Motor coordination & 8,896 & 65,853 \\
6 & Static power & 6,920 & 72,773 \\
\hline
\end{tabular}

Table 4. Matrix of actorial analysis of 12 yrs boys' testing. Rotation method: Varimax with Kwiser's normalization

\begin{tabular}{|c|c|c|c|c|c|c|}
\hline \multirow{2}{*}{$\begin{array}{l}\text { № } \\
\text { № }\end{array}$} & \multirow{2}{*}{ Description of parameters } & \multicolumn{4}{|c|}{ Компонента } & \multirow{2}{*}{ h2 } \\
\hline & & 1 & 2 & 3 & 4 & \\
\hline 1 & Height, $\mathrm{cm}$ & & & & ,821 & ,781 \\
\hline 2 & Body mass, $\mathrm{kg}$ &,- 364 & & & ,703 & 719 \\
\hline 3 & $\mathrm{VCL}, \mathrm{cm} 3$ & & ,533 & & ,511 & ,588 \\
\hline 4 & Right hand dynamometry, kg & & ,776 & ,300 & & 768 \\
\hline 5 & Left hand dynamometry, kg & & ,729 & & & 659 \\
\hline 6 & Pressing ups, times & ,632 & & ,431 & & ,687 \\
\hline 7 & Chin ups, times & ,761 & & ,441 & & ,842 \\
\hline 8 & Legs' rising, hanging on Sweden wall, times & ,654 & &, 525 & & ,783 \\
\hline 9 & Angle on parallel bars, sec., times & ,455 & ,309 & ,665 & & ,761 \\
\hline 10 & Torso rising from position lying on back during 1 minute, times & ,806 & ,354 & & & ,803 \\
\hline 11 & Forward torso bending in sitting position (legs apart), $\mathrm{cm}$ &,- 513 &,- 609 & & & 707 \\
\hline 12 & Hanging on bent arms, sec & ,549 & & 629 & & ,788 \\
\hline 13 & Torso rising from lying on abdomen position during $30 \mathrm{sec}$., times & ,696 & & ,433 & & ,723 \\
\hline 14 & Long jump from the spot, $\mathrm{cm}$ & & & 699 & ,328 & ,613 \\
\hline 15 & Throw of filled ball (1 kg) from sitting position, $\mathrm{cm}$ & 341 & & & ,712 & ,640 \\
\hline 16 & Shuttle run, $4 \times 9 \mathrm{~m}$, sec. &,- 596 & ,438 &,- 371 & & 708 \\
\hline 17 & Forward roll, level of mastering & ,887 & & & & ,812 \\
\hline 18 & Backward roll, level of mastering & 927 & & & & ,902 \\
\hline 19 & Vault, level of mastering & ,806 &,- 315 & & & ,772 \\
\hline 20 & Climbing rope (three attempts), level of mastering & ,858 & & & & ,778 \\
\hline 21 & Bridge, level of mastering &, 572 &,- 547 & ,309 & & ,762 \\
\hline 22 & Stance on shoulder blades, level of mastering & 860 & & & & 817 \\
\hline
\end{tabular}


Table 5. Full explained dispersion

\begin{tabular}{|c|c|c|c|}
\hline \multirow{2}{*}{ Component } & \multirow{2}{*}{ Interpretation } & \multicolumn{2}{|c|}{ Sum of squares of rotation loads } \\
\hline & & $\%$ of dispersion & Cumulative \% \\
\hline 1 & Level of mastering and motor fitness & 36,646 & 36,646 \\
\hline 2 & Physical condition & 14,067 & 50,713 \\
\hline 3 & Speed power & 12,639 & 63,352 \\
\hline 4 & Physical condition & 11,255 & 74,607 \\
\hline
\end{tabular}

Table 6. Matrix of actorial analysis of 13 yrs boys' testing. Rotation method: Varimax with Kwiser's normalization

\begin{tabular}{|c|c|c|c|c|c|c|c|}
\hline \multirow{2}{*}{$\begin{array}{l}\text { № } \\
\text { № }\end{array}$} & \multirow{2}{*}{ Description of parameters } & \multicolumn{5}{|c|}{ Components } & \multirow{2}{*}{$h^{2}$} \\
\hline & & 1 & 2 & 3 & 4 & 5 & \\
\hline 1 & Height, cm & & & ,706 & & & 656 \\
\hline 2 & Body mass, kg &,- 380 & & ,762 &,- 357 & & ,856 \\
\hline 3 & $\mathrm{VCL}, \mathrm{cm} 3$ & & ,381 & ,501 &,- 486 & & 668 \\
\hline 4 & Right hand dynamometry, kg & & & ,937 & & & 901 \\
\hline 5 & Left hand dynamometry, kg & & & ,950 & & & ,921 \\
\hline 6 & Pressing ups, times & ,424 & ,666 & & & 314 & ,737 \\
\hline 7 & Chin ups, times & ,388 & 699 & & & & 810 \\
\hline 8 & Legs' rising, hanging on Sweden wall, times & ,345 & ,766 & & & & ,795 \\
\hline 9 & Angle on parallel bars, sec., times & & 791 & & & & ,792 \\
\hline 10 & $\begin{array}{l}\text { Torso rising from position lying on back during } 1 \text { minute, } \\
\text { times }\end{array}$ & ,376 & 680 & & & & 800 \\
\hline 11 & Forward torso bending in sitting position (legs apart), cm & & &,- 325 & ,821 & & 813 \\
\hline 12 & Hanging on bent arms, sec & & ,728 & & & ,380 & ,858 \\
\hline 13 & $\begin{array}{l}\text { Torso rising from lying on abdomen position during } 30 \\
\text { sec., times }\end{array}$ & ,697 & ,569 & & & & 814 \\
\hline 14 & Long jump from the spot, $\mathrm{cm}$ & 347 & ,582 & & & & ,595 \\
\hline 15 & Throw of filled ball (1 kg) from sitting position, $\mathrm{cm}$ & ,334 & & & & ,796 & 797 \\
\hline 16 & Shuttle run, $4 \times 9 \mathrm{~m}$, sec. & & & & & ,463 & ,260 \\
\hline 17 & Forward roll, level of mastering & ,904 & & & & & ,883 \\
\hline 18 & Backward roll, level of mastering & ,889 & & & & & ,858 \\
\hline 19 & Vault, level of mastering & ,886 & & & & & ,895 \\
\hline 20 & Climbing rope (three attempts), level of mastering & ,881 & & & & & ,876 \\
\hline 21 & Bridge, level of mastering & ,794 & & & & & 822 \\
\hline 22 & Stance on shoulder blades, level of mastering & ,909 & & & & & ,917 \\
\hline
\end{tabular}

Table 7. Full explained dispersion

\begin{tabular}{|c|c|c|c|}
\hline \multirow{2}{*}{ Component } & \multirow{2}{*}{ Interpretation } & \multicolumn{2}{|c|}{ Sum of squares of rotation loads } \\
\hline & & $\%$ of dispersion & Cumulative \% \\
\hline 1 & Level of mastering and motor fitness & 28,497 & 28,497 \\
\hline 2 & Strength of abdomen mucles & 20,299 & 48,796 \\
\hline 3 & Physical condition & 16,126 & 64,922 \\
\hline 4 & Flexibility & 6,994 & 71,916 \\
\hline 5 & Speed power & 6,821 & 78,737 \\
\hline
\end{tabular}

sitting position, cm"., "Shuttle run $4 \times 9 \mathrm{~m}$, sec".

The level of mastering the exercises "Forward roll", "Backward roll", "Vault", "Climbing rope (three attempts)", "Bridge", "Stance on shoulder blades" statistically confidently increases with age $(p<0,001)$ (see table 1).

Thus, in 11-13 yrs age boys we noted positive dynamic of physical condition, motor abilities and gymnastic exercises' mastering level indicators.

For specifying motor fitness influence on physical exercises' mastering level we fulfilled factorial analysis.

Results of factorial analysis are given in tables 1-7. Inthe process of analysis in $11 \mathrm{yrs}$ boys we marled out six factors, which explain $72,773 \%$ of total dispersion (see table 2-3).

Factor 1 has the highest weight $(21,735 \%)$ and 
correlates with the following tests' results:

- Climbing rope (three attempts), level of mastering.

- Vault, level of mastering,834.

- $\quad$ Backward roll, level of mastering, 778.

The factor was named level of mastering and motor fitness.

Factor 2 has weight 17,108\% and correlates with the follwing results:

- Height, cm, 778.

- $\quad$ Body mass, kg, ,702.

The factor was named physical condition.

Factor 3 has weight 13,390\% and correlates with the following:

- $\quad$ Right hand dynamometry, kg - -,,606.

- Left hand dynamometry, $\mathrm{kg}$ - -,693.

The factor was named hand's strength.

Factor 4 has weight $8,954 \%$ and correlates with the followig:

- VCL, $\mathrm{cm}^{3}-, 741$.

The factor was named functional state of respiratory system.

Factor 5 has weight 8,896\% and correlates:

- Forward torso bending in sitting position (legs apart), $\mathrm{cm}-, 635$.

- $\quad$ Shuttle run, 4x9 m, sec.- 615 .

The factor was named motor coordination.

Factor 6 has weight $6,920 \%$ and correlates with the following tests' results:

Hanging on bent arms, sec.- - $\mathbf{4 4 9}$.

The factor was named static power.

- Analysis of communities showed that in motor fitness structure of 11 yrs boys the biggest influence is rendered by: "Chin ups— ,897"; "Climbing rope (three attempts), level of mastering.

— 860; "Hanging on bent arms, — ,854"; "Vault, level of matsrerig — , ,815".

Analyzing 12 yrs boys we marked out 4 factors, which explain $74,607 \%$ of total indicators' dispersion (see table 4, 5).

Factor 1 has the highest weight $(36,646 \%)$ and correlates with: .

- Backward roll, level of mastering — ,927.

- Forward roll, level of mastering- ,887.

- Climbing rope (three attempts), level of mastering , 858 .

- Vault, level of matsrerig_, 806 .

The factor was named level of mastering and motor fitness.

Factor 2 has weight - 14,067\% and correlates with physical condition indicatotrs:

- $\quad$ Right hand dynamometry, $\mathrm{kg}-\mathbf{, 7 7 6}$.

- $\quad$ Left hand dynamometry, kg- ,729.

- VCL, $\mathrm{cm}^{3}$ -, 533 .

The factor was named physical condition.

Factor 3 has weight - 12,639\% and correlates with the following:

- $\quad$ Long jump from the spot, $\mathrm{cm}-\mathbf{6 9 9 9}$.

- Angle on parallel bars, sec- $\mathbf{6 6 5}$.

- Hanging on bent arms, sec.- ,629.
The factor was namedspeed power

Factor 4 has weight - 11,255\% and correlates:

Height, $\mathrm{cm}-\mathbf{8 2 1}$.

- Body mass,-, ,703.

The factor was named physical condition.

Analysis of communities showed that in motor fitness structure of $12 \mathrm{yrs}$ boys the beggest influence is rendered by: "Backward roll, level of mastering — ,902"; "Chin ups, times - ,842"; "Forward roll, level of mastering ,812”.

Analyzing 13 yrs boys we marked out 5 factors, which explain $78,737 \%$ of total indicators' dispersion (see table $6,7)$.

Factor 1 has the biggest weight $(28,497 \%)$.and correlates with the following results:

- Forward roll, level of mastering - ,904.

- Backward roll, level of mastering - , $\mathbf{8 8 9}$.

- Vault, level of matsrerig - , $\mathbf{8 8 6}$.

- Climbing rope (three attempts), level of mastering ,881.

The factor was named level of mastering and motor fitness.

Factor 2 has the biggest weight - 20,299\% and correlates with the following results:

- Angle on parallel bars, sec — ,791.

- Leggs' rising on Sweden wall, times- ,766.

- Hanging on bent arms, sec- ,728.

The factor was named strength og abdomen muscles.

Factor 3 has weight- 16,126\% and correlates with the following:

- Left hand dynamometry, kg-,950.

- $\quad$ Right hand dynamometry, $\mathrm{kg}$ — ,937.

- Body mass, kg — ,762.

- Height, cm- ,706.

The factor was named physical condition.

Factor 4 has weight $-6,994 \%$ and correlates with the following results:

- Forward torso bending in sitting position (legs apart), $\mathrm{cm}-, 821$.

The factor was named flexibility.

Factor 5 has weight $-6,821 \%$ and correlated with the following tests' results:

Throw of filled $(1 \mathrm{~kg})$ ball from sitting position, $\mathrm{cm}$ - ,796.

The factor characterizes speed power.

Analysis of communities showed that motor fitness of 13 yrs. boys is influenced to the largest extent: "Left hand dynamometry, $\mathrm{kg}$ — ,921"; "Vault, level of mastering,895"; "Stance on shoulder blades, level of mastering,917; "Forward roll, level of mastering — ,883".

\section{Discussion}

In our work we studied assumption about wholeness of motor abilities processes'development and training from prosition of systemic approach [3,8]. We found that variation of results in total dispersion of 11-13 yrs boys by $72,773 \%, 74,607 \%, 78,737 \%$ depends on the regarded factors. Mastering level in factorial structure has weight $17,505 \%$ (11 years), 36,646\% (12yeasr), 28,497\% (13 
years). Alnysis of communities showed that in 11-13 yrs boys develppment of motor abilities is effective is they are a component of the mastered motor skills.

The same dynamic of physical exercises mastering we observed in 11-13 yrs girls. We found that variation of results in total dispersion of $11-13$ yrs girls by $81,259 \%$, $79,353 \%, 71,019 \%$ is conditioned by the following factors: physical condition, level of motor abilities and level of physical exercises' mastering. In factorial structure level of physical exercises' mastering has weight 16,435\% (11 years), $27,963 \%$ (12 years), $17,010 \%$ (13 years) [31].

In 11-13 yrs boys we observed higher contribution of mastering level in motor fitness structure.

The presented data supplements the results of Xu X. and Ke F. [30], Repko E. et al. [10], Khudolii O.M. et al. [28].

The conducted factorial analysis permitted to regard development of motor abilities and training as holistic process. It supplements the data of Ivashchenko O. et al. [21], Ivashchenko O. et al. [22] about effectiveness of factorial analysis application in physical education. Analysis of communities in factorial analysis permits to find the role of one or another indicator in factorial structure of the process. It points at demand in application of multidimensional mathematical statistic methods in studing of children's and adolescents' physical education laws $[8,24,25,33]$.

\section{Conclusions}

In 11-13 yrs boys we found positive dynamic of physical condition, indicators of motor abilities' development and mastering level of gymnastic exercises.

We found that variation of results in total dispersion of $11-13$ yrs boys by $72,773 \%, 74,607 \%, 78,737 \%$ depends on the regarded factors. Mastering level in factorial structure has weight $17,505 \%$ (11 years), 36,646\% (12yeasr), 28,497\% (13 years). Analysis of communities in factorial analysis permits to find that in 11-13 yrs boys development of motor abilities is effectice if they are a component of the mastered mototr skills.

\section{Acknowledgements}

The study has been fulfilled in compliance with plan of scientific research works of Ministry of education and science, youth and sports of Ukraine by topic 13.04. "Modeling of training process and motor abilities' development in children and adolescents" (2013-2014) (state registration number 0113U002102).

\section{Conflict of interests}

The authors declare that there is no conflict of interests.

\section{References}

1. Bodnar I. Integrative physical education of different health groups'schoolchildren. Lviv: LSUPC; 2014. (in Ukrainian)

2. Vas'kov IuV. Innovative approaches to organization of comprehensive educational establishments' pupils physical education. Teoria ta metodika fizichnogo vikhovannia, 2016;4:5-12. doi:10.17309/tmfv.2016.4.1174 (in Ukrainian)

3. Vlasov A, Demichkovs'kij A, Ivashchenko O, Lopat'iev A, Pitin M, P'ianilo Ia, Khudolii O. Systemic approach and mathematical modeling of biological and natural objects and processes. Fiziko-matematichne modeliuvannia ta informacijni tekhnologii, 2016;23:17-28. (in Ukrainian)

4. Ivashchenko OV, Shepelenko GP. Comparative characteristics of Coordination fitness and power of middle class. Teoria ta metodika fizichnogo vikhovannia, 2014;2:2230. doi:10.17309/tmfv.2014.2.1096 (in Ukrainian)

5. Ivashchenko OV, Mushketa R, Khudolii OM, Iermakov SS. Characteristic force preparedness boys 6-7 grades. Teoria ta metodika fizichnogo vikhovannia, 2014;3:17-24. doi:10.17309/tmfv.2014.3.1104 (in Ukrainian)

6. Ivashchenko OV, Pashkevich SA, Krinin IuV. Comparative characteristics of functional coordination and force readiness boys $8-9$ grades. Teoria ta metodika fizichnogo vikhovannia, 2014;2:31-39. doi:10.17309/tmfv.2014.2.1099 (in Ukrainian)

7. Ivashchenko OV, Ceslicka M, Khudolii OM, Iermakov SS. Modeling power fitness girls grades 6-7. Teoria ta metodika fizichnogo vikhovannia, 2014;3:10-16. doi:10.17309/ tmfv.2014.3.1103 (in Ukrainian)

8. Ivashchenko OV. Modelling of physical education students: Monograph. Kharkiv: OVS; 2016. (in Ukrainian)

9. Krucevich T, Trachuk S, Napadij A. Planning of physical culture training process for secondary comprehensive schools' pupils. Teoriia i metodika fizichnogo vikhovannia $i$ sportu, 2016;1:36-42. (in Ukrainian)

10.Repko E, Kozin S, Kostyrko A. Training of pre school age and junior school age children to motor actions on the base of their psychological and physical characteristics on example of rock climbing. Zdorov'e, sport, reabilitaciia, 2016; 2:4650. (in Ukrainian)

11. Khudolii OM, Ivashchenko OV. Features functional coordination force preparedness and girls grades 7-8. Teoria ta metodika fizichnogo vikhovannia, 2014;2:15-21. doi:10.17309/tmfv.2014.2.1095

12.Al-Ravashdeh Abdel Baset, Kozina ZL, Bazilyuk TA, Ilnickaya AS. Methodic of senior pupils' training to throwing movements on the bases of technology of complex impact on motor and intellectual development. Pedagogics, psychology, medical-biological problems of physical training and sports, 2015. 19 (11), 3-10. doi:10.15561/18189172.2015.1101

13.Al-Ravashdeh Abdel Baset, Kozina Zh.L., Bazilyuk T.A., Ilnickaya, A.S. Influence of motor skills' training methodic on senior pupils' speed-power and endurance qualities at light athletic trainings with aplication of interdisciplinary connections. Pedagogics, psychology, medical-biological problems of physical training and sports, 2015; 19(10):3-10. doi:10.15561/18189172.2015.1001

14.Altunsoz IH, Goodway JD. Skiping to motor competence: the influence of project successful kinesthetic instruction for preschoolers on motor competence of disadvantaged preschoolers. Physical Education and Sport Pedagogy, 2016;21(4): 366-385. doi:10.1080/17408989.2015.1017453

15.Arziutov G, Iermakov S, Bartik P, Nosko M, Cynarski WJ. The use of didactic laws in the teaching of the physical elements involved in judo techniques. Ido Movement for Culture, 2016;16(4):21-30. doi:10.14589/ido.16.4.4

16.Chatzipanteli A, Digelidis N, Karatzoglidis C, Dean R. A tactical-game approach and enhancement of metacognitive 
behaviour in elementary school students. Physical Education and Sport Pedagogy, 2016;21(2):169-184. doi:10.1080/174 08989.2014.931366

17.Chernenko SO. Effectieness of junior form pupils' training of gymnastic exercises in different modes of their fulfillment. Pedagogics, psychology, medical-biological problems of physical training and sports, 2015;19(8):65-74. doi:10.15561/18189172.2015.0809

18.Cieślicka M, Ivashchenko O. Features of formation of the cumulative effect of power loads in boys 7 years old. Journal of Education, Health and Sport, 2017;7(1):198-208. doi:10.5281/zenodo. 250599

19.Darnis F, Lafont L. Cooperative learning and dyadic interactions: two modes of knowledge construction in socioconstructivist settings for team-sport teaching. Physical Education and Sport Pedagogy, 2015;20(5):459-473. doi:1 0.1080/17408989.2013.803528

20.Ivashchenko OV, Kapkan OO. Simulation of process of 14 15 years old girls' training of light athletic and gymnastic exercises. Pedagogics, psychology, medical-biological problems of physical training and sports, 2015;19(8):32-39. doi:10.15561/18189172.2015.0805

21.Ivashchenko O, Khudolii O, Yermakova T, Iermakov S, Nosko M, Nosko Y. Factorial and discriminant analysis as methodological basis of pedagogic control over motor and functional fitness of 14-16 year old girls. Journal of Physical Education and Sport, 2016;16(2):442 - 451. doi:10.7752/ jpes.2016.02068

22.Ivashchenko O, Khudolii O, Iermakov S, Lochbaum MR, Cieslicka M, Zukow W, et al. Intra-group factorial model as the basis of pedagogical control over motor and functional fitness dynamic of 14-16 years old girls. Journal of Physical Education and Sport, 2016;16(4):1190 - 1201

23.Ivashchenko O, Cieślicka M. Features of evaluations of power loadsin boys 7 years old. Journal of Education, Health and Sport, 2017;7(1):175-183. doi:10.5281/zenodo.249184

24.Ivashchenko OV. Methodic of pedagogic control of 1617 years' age girls' motor fitness. Pedagogics, psychology, medical-biological problems of physical training and sports,
2016;5:26-32. doi:10.15561/18189172.2016.0504

25.Ivashchenko OV, Kapkan OO. Informative pedagogic control indicators of 14-15 years age girls' motor fitness. Pedagogics, psychology, medical-biological problems of physical training and sports, 2016;6:18-25. doi:10.15561/18189172.2016.0603

26.Kapkan OO. Features of 14-15 years' age boys' training to physical exercises. Pedagogics, psychology, medicalbiological problems of physical training and sports, 2015;19(9):26-32. doi:10.15561/18189172.2015.0904

27.Khudolii OM. Theoretical-methodic principles of system of junior, 7-13 yrs. age, gymnasts' training Dokt. Diss., Kiev; 2011. (in Ukrainian)

28.Khudoli, OM, Ivashchenko OV, Chernenko SO. Simulation of junior shcoolchildren's training to acrobatic exercises and vaults. Pedagogics, psychology, medical-biological problems of physical training and sports, 2015;19(7):64-71. doi:10.15561/18189172.2015.0709

29.Khudolii OM, Ivashchenko OV, Iermakov SS, Rumba OG. Computer simulation of junior gymnasts' training process. Science of Gymnastics Journal, 2016;8;(3):215-228.

30.Xu X, Ke F. From psychomotor to 'motorpsycho': Learning through gestures with body sensory technologies. Educational Technology Research and Development, 2014;62;(6):711741. doi:10.1007/s11423-014-9351-8

31.Ivashchenko OV. Special aspects of motor fitness influence on level of 11-13 years' age girls' physical exercises' mastering. Pedagogics, psychology, medical-biological problems of physical training and sports, 2017;21(1), 11-17. doi:10.15561/18189172.2017.0102

32.Ivashchenko OV. Classification of 11-13 yrs girls' motor fitness, considering level of physical exercises' mastering. Pedagogics, psychology, medical-biological problems of physical training and sports, 2017;21(2), 65-70. doi:10.15561/18189172.2017.0203

33.Lopatiev A, Ivashchenko O, Khudolii O, Pjanylo Y, Chernenko S, Yermakova T. Systemic approach and mathematical modeling in physical education and sports. Journal of Physical Education and Sport, 2017;17 (1), 146155.

\section{Information about the authors:}

Ivashchenko O.V.; http://orcid.org/0000-0002-2708-5636; tmfv@tmfv.com.ua; H.S. Skovoroda Kharkiv National Pedagogical University; Alchevskyh str. 29, Kharkiv, 61002, Ukraine.

lermakov S.S.; http://orcid.org/0000-0002-5039-4517; sportart@gmail.com; Kazimierz Wielki University; Sport str. 2, of.209, 85-064 Bydgoszcz, Poland.

Khudolii O.M.; http://orcid.org/ 0000-0002-5605-9939; tmfv@tmfv.com.ua; H.S. Skovoroda Kharkiv National Pedagogical University; Alchevskyh str. 29, Kharkiv, 61002, Ukraine.

Cretu Marian; http://orcid.org/0000-0003-1934-0534; marian.cretu@efsupit.ro; University of Pitesti: Pitesti, arges, Romania; Str. Targul din Vale, nr.1, 110040 Pitesti, Arges, Romania.

Potop Vladimir; http://orcid.org/0000-0001-8571-2469; vladimir_potop@yahoo.com; Ecological University of Bucharest; Bulevardul General Vasile Milea 1G, București 061341, Romania.

Cite this article as: Ivashchenko OV, Iermakov SS, Khudolii OM, Cretu M, Potop V. Level of physical exercises' mastering in structure of 11-13 yrs age boys' motor fitness. Pedagogics, psychology, medical-biological problems of physical training and sports, 2017;21(5):236-243. doi:10.15561/18189172.2017.0506

The electronic version of this article is the complete one and can be found online at: http://www.sportpedagogy.org.ua/index.php/PPS/issue/archive

This is an Open Access article distributed under the terms of the Creative Commons Attribution License, which permits unrestricted use, distribution, and reproduction in any medium, provided the original work is properly cited (http://creativecommons.org/licenses/by/4.0/deed.en).

Received: 11.06 .2017

Accepted: 05.07.2017; Published: 25.09.2017 